\title{
Huntington's Disease Clinical Trials Corner: June 2019
}

\author{
Filipe B. Rodrigues ${ }^{\mathrm{a}, \mathrm{b}, \mathrm{c}}$, Joaquim J. Ferreira ${ }^{\mathrm{b}, \mathrm{c}, \mathrm{d}}$ and Edward J. Wild ${ }^{\mathrm{a}, *}$ \\ ${ }^{a}$ UCL Huntington's Disease Centre, UCL Queen Square Institute of Neurology, University College \\ London, London, $U K$ \\ ${ }^{\mathrm{b}}$ Laboratory of Clinical Pharmacology and Therapeutics, Faculdade de Medicina, Universidade \\ de Lisboa, Lisbon, Portugal \\ ${ }^{\mathrm{c}}$ Instituto de Medicina Molecular, Lisbon, Portugal \\ ${ }^{\mathrm{d}}$ CNS - Campus Neurológico Sénior, Torres Vedras, Portugal
}

\begin{abstract}
In this edition of the Huntington's Disease Clinical Trials Corner we expand on the HD-DBS and on the TRIHEP3 trials, and we list all currently registered and ongoing clinical trials in Huntington's disease.
\end{abstract}

Keywords: Huntington disease, clinical trials

\section{INTRODUCTION}

The Huntington's Disease Clinical Trials Corner is a regular section devoted to highlighting ongoing and recently completed clinical trials in Huntington's disease (HD). Clinical trials previously reviewed by the Huntington's Disease Clinical Trials Corner are listed in Table 1.

In this edition, we highlight the HD-DBS trial (NC T02535884)(1), and the TRIHEP3 trial (NCT0245 3061)(2). We tabulate all currently registered and ongoing clinical trials in Tables 2 to 4 . For further details on the methodology used, please refer to the first edition of Huntington's Disease Clinical Trials Corner(3).

If you would like to draw attention to specific trials, please feel free to email us at: f.rodrigues@ucl.ac.uk and e.wild@ucl.ac.uk.

In addition to the above, the published report of the IONIS-HTT $\mathrm{Rx}_{\mathrm{R}}$ trial (NCT02519036) is worthy of mention. The paper reports that monthly intrathecal IONIS-HTT ${ }_{\mathrm{Rx}} /$ RG6042 - an antisense oligonucleotide that signals wild-type and mutant

\footnotetext{
*Correspondence to: Edward J. Wild, UCL Huntington's Disease Centre, Russell Square 10-12, London, WC1B 5EH, UK. E-mail: e.wild@ucl.ac.uk.
}

huntingtin pre-mRNA to be degraded by RNase H1 - was safe and well-tolerated, and produced dosedependent reductions in cerebrospinal fluid mutant huntingtin in early HD patients (4). This is an interesting signal but caution should be exercised as to whether this reduction translates into a clinically significant benefit for people with HD. Further investigation into the effects of this drug are expected from the currently ongoing phase 3 GENERATION-HD1 trial (NCT03761849)(5) and associated studies (6-10). These studies will help us better characterize the safety profile of this compound, define the most efficient posology, and understand if it is associated with a clinically relevant benefit, and towards which disease domain.

\section{ONGOING CLINICAL TRIALS}

A list of all ongoing clinical trials is given in Tables 2-4.

HD-DBS (NCT02535884)

\section{Study title}

Deep Brain Stimulation (DBS) of the Globus Pallidus (GP) in Huntington's Disease (HD) (HDDBS)(1). 
Table 1

Clinical trials previously reviewed by the Huntington's Disease Clinical Trials Corner

\begin{tabular}{llll}
\hline & Trial name & Intervention & Edition \\
\hline NCT02519036 & IONIS-HTTRx & IONIS-HTT ${ }_{\text {Rx }}{ }^{*}$ & September 2017(3) \\
NCT02215616 & LEGATO-HD & Laquinimod & \\
NCT02197130 & Amaryllis & PF-02545920 & \\
NCT02006472 & PRIDE-HD & Pridopidine & \\
NCT03225833 & PRECISION-HD1 & WVE-120101 & February 2018(28) \\
NCT03225846 & PRECISION-HD2 & WVE-120102 & \\
NCT01795859 & FIRST-HD & Deutetrabenazine & \\
NCT02481674 & SIGNAL & VX15/2503 & August 2018(29) \\
NCT00712426 & CREST-E & Creatine & \\
NCT03761849 & GENERATION-HD1 & RG6042* & January 2019(30) \\
NCT03344601 & PACE-HD & Physical activity & \\
NCT02535884 & HD-DBS & Deep brain stimulation & June 2019 \\
NCT02453061 $^{*}$ TONIS-HTT & \\
${ }_{\text {IOx }}$ and & RG6042 refer to the same molecule. &
\end{tabular}

\section{Intervention}

DBS of the GP (11) with Medtronic ACTIVA® PC neurostimulator (Model 37601).

\section{Description}

The HD-DBS trial, sponsored by Heinrich-Heine University, aims to evaluate the efficacy and safety of pallidal DBS in adults (18 or more years of age) with manifest HD (i.e. clinically symptomatic and genetically confirmed $[\mathrm{CAG} \geq 36]$ ) and moderate disease stage (defined by the investigators as an Unified Huntington's Disease Rating Scale [UHDRS] total motor score $[$ TMS] $\geq 30$ ), chorea (UHDRS chorea score $\geq 10$ ) and a Mattis Dementia Rating Scale $\geq 120$, comparing with sham stimulation, for motor function.

People with juvenile or predominantly bradykinetic forms of the disease, postural instability, unstable medication in the 6 weeks previous to inclusion, unstable medical or psychiatric comorbidities, coagulopathies and/or increased risk of haemorrhage, implanted pacemaker or defibrillator, pregnant or breast-feeding are not eligible for this study.

This trial is an international, multi-centre, randomized, sham-controlled, double-blind, parallel study. It has 2 study arms: the stimulation group, where participants have stimulation turned on immediately after implantation of the stimulator; and the sham stimulation group, where participants will have a stimulator implanted but it will not be switched on. The study lasts 12 weeks, and after that period all participants' stimulators will be turned on.

The trial has already started recruitment, and has a recruitment target of 50 participants, over 4 countries (Austria, France, Germany and Switzerland) and 12 sites.
The primary outcome is the UHDRS TMS at 12 weeks, measured as the difference in the mean change from baseline between the stimulation arm and the sham-stimulation arm. The secondary outcomes include the UHDRS chorea score and the bradykinesia items, the Burke-Fahn-Marsden Dystonia Rating Scale, the Q-Motor choreomotography task, the Mattis Dementia Rating Scale, the Verbal Fluency Test, the Symbol Digits Modalities Test, the Stroop Test, the Hospital Anxiety and Depression Scales and the Snaith Irritability Scale, the Problem Behaviours Assessment Short Form, the Short Form 36 Health Survey, the Clinical Global Impression Scale, and safety.

\section{Sponsors/funders}

Heinrich-Heine University, KKS Netzwerk, Medtronic, the George Huntington Institute, EHDN and CHDI Foundation.

\section{Comments}

DBS is a relatively well-studied intervention for some manifestations of Parkinson's disease, tremor and dystonia. DBS involves the surgical implantation of electrical electrodes in the deep brain structures, connected via a wire to an implantable pulse generator (i.e. stimulator) usually positioned subcutaneously in the pectoral region. Although the precise mechanisms of action are still not completely understood, this intervention is aimed at interrupting certain neuronal circuits.

In Parkinson's disease DBS is frequently used to minimize levodopa-induced dyskinesia, which has a similar phenomenology to chorea in HD, but a different aetiology. This is accomplished by bet- 


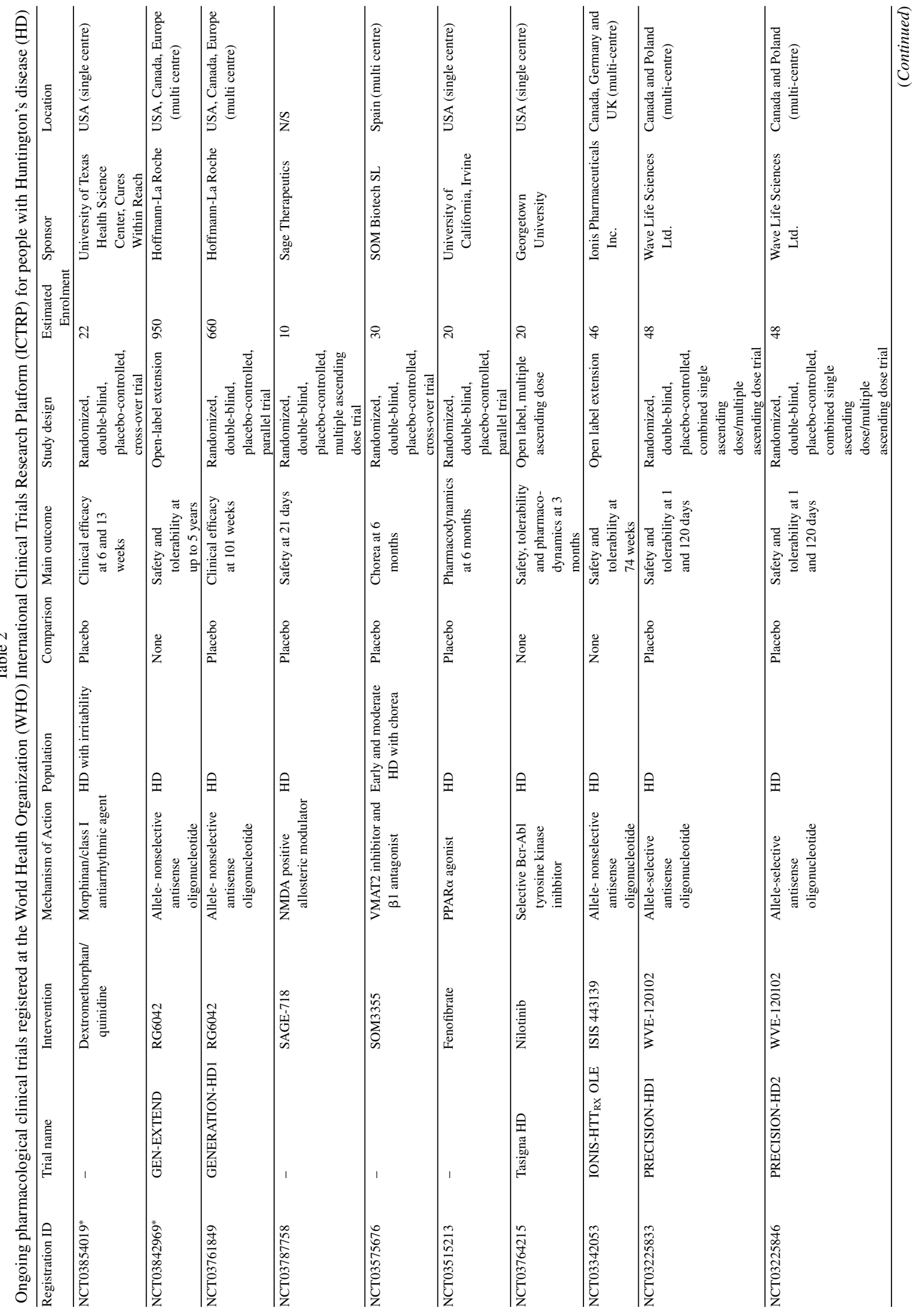




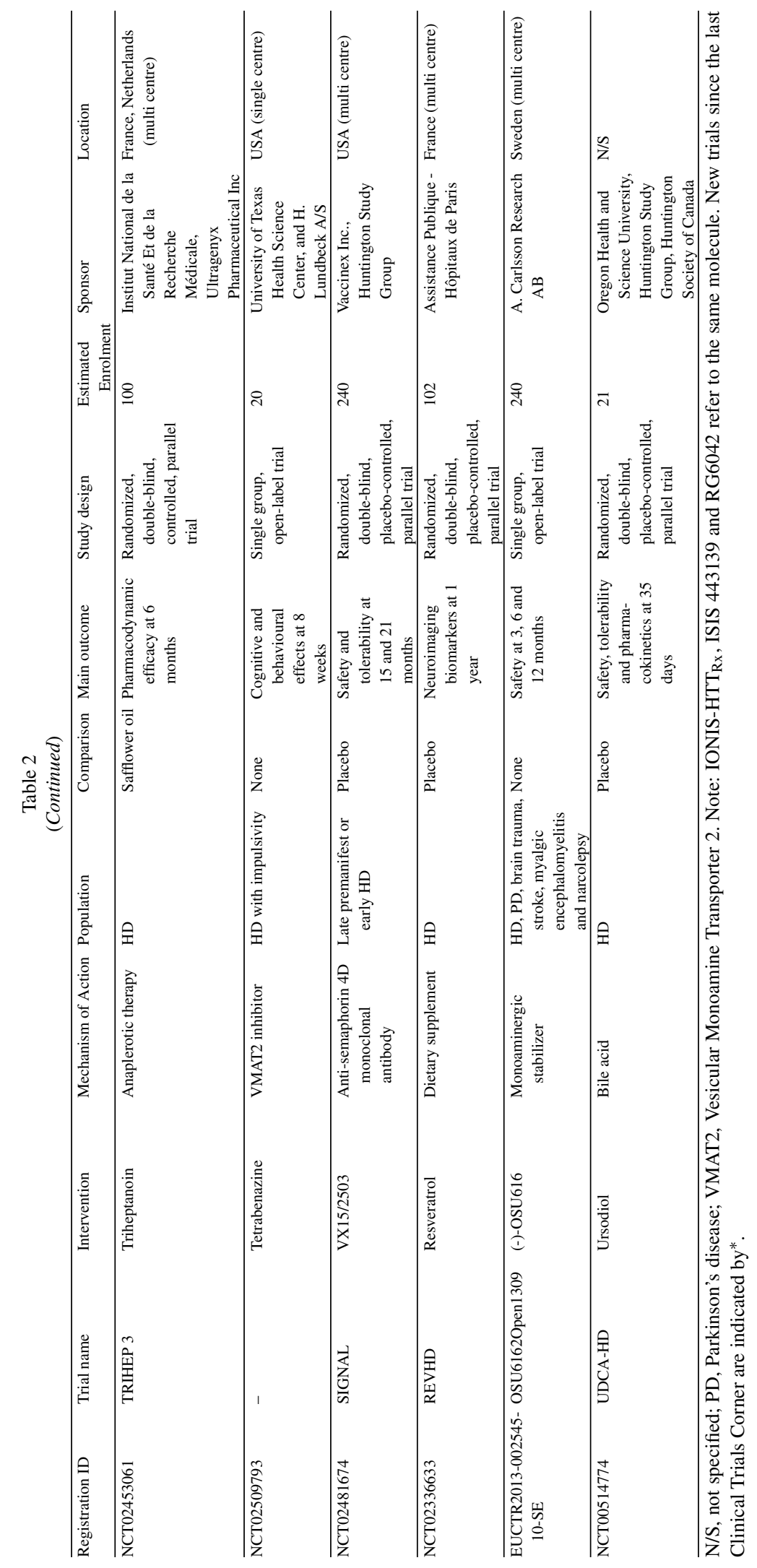




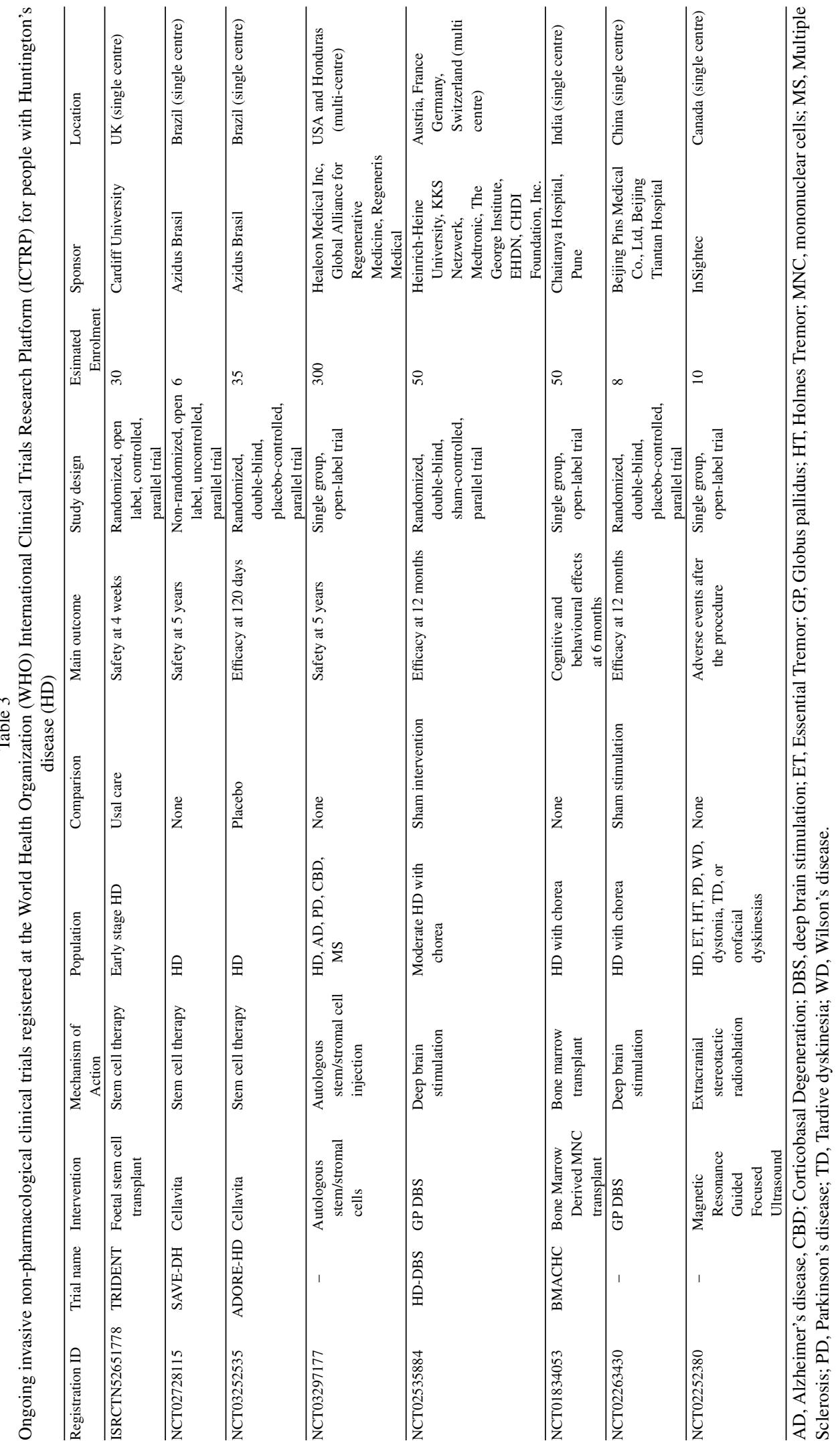




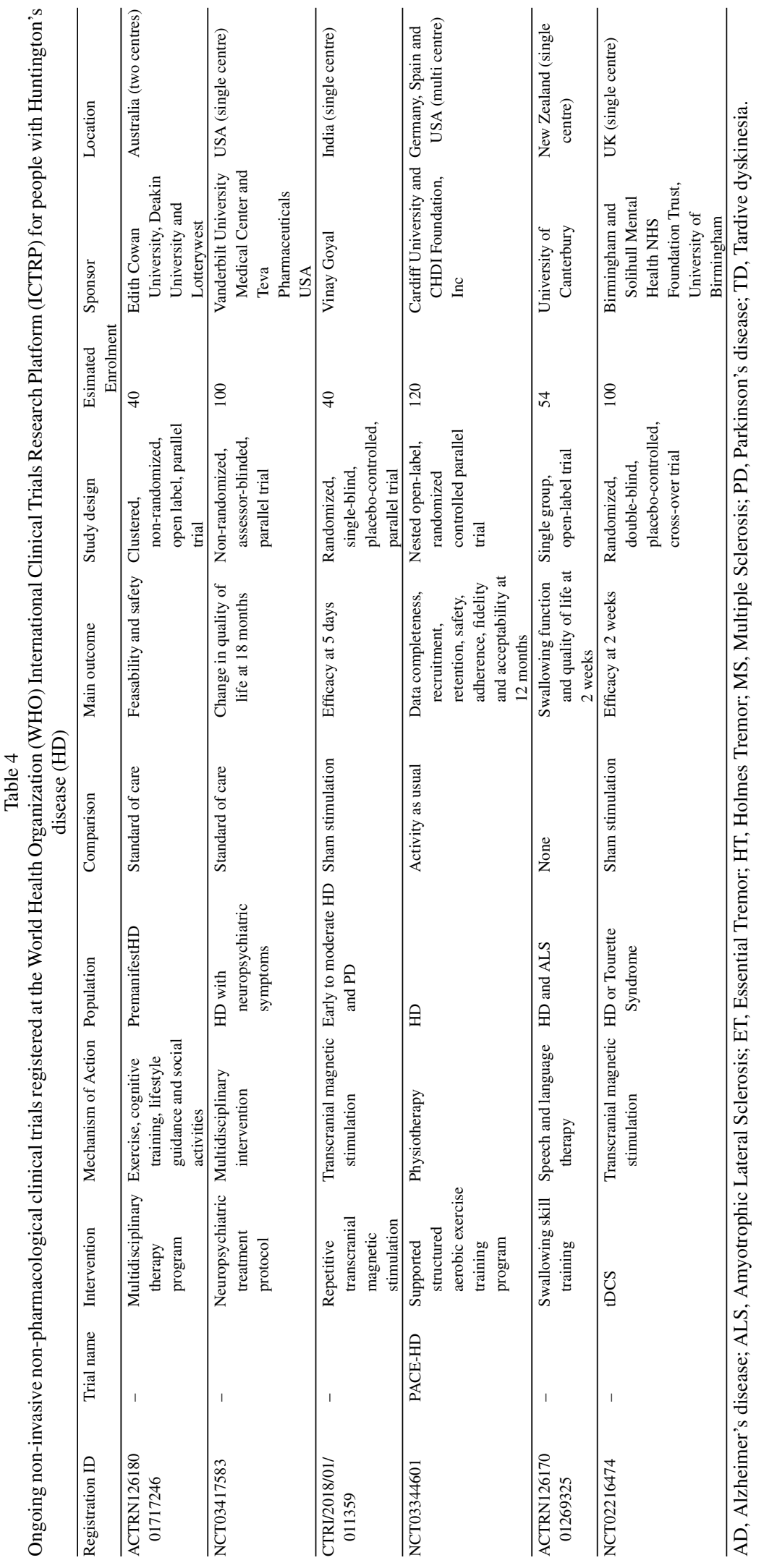


ter controlling the cardinal features of PD, hence reducing the levodopa dose equivalents. There is still uncertainty about whether there is a direct effect of DBS over dyskinesia. In HD, chorea is thought to be caused by the loss of striatal projections to the indirect basal ganglia pathway and consequent thalamic overactivity (11), and although there is a shortage of good-quality evidence, some pilot studies have shown interesting preliminary results when manipulating these circuits with DBS in $\operatorname{HD}(12,13)$.

The scarcity of data so far accumulated precludes drawing conclusions on the safety and efficacy profile of this intervention in HD, but it seems sensible to assume that this population may be susceptible to the same intervention-related adverse events as other tested populations. Disease-specific side effects may be more difficult to predict.

Only large well-controlled prospective controlled studies will allow us to fully understand the efficacy and safety profile of DBS in HD.

\section{TRIHEP3 (NCT02453061)}

\section{Study title}

A Comparative Phase 2 Study Assessing the Efficacy of Triheptanoin, an Anaplerotic Therapy in Huntington's Disease (TRIHEP3)(2).

\section{Intervention}

Triheptanoin oil $1 \mathrm{~g} / \mathrm{kg} /$ day (14).

\section{Description}

The TRIHEP3 trial, sponsored by the Institut National de la Santé et de la Recherche Médicale and Ultragenyx Pharmaceutical Inc., aims to evaluate the effects of daily triheptanoin in adults $(\geq 18$ years of age) with genetically confirmed manifest HD (i.e. UHDRS TMS between 5 and 40), compared with daily safflower oil. People with a BMI $<18$ or $>30$, hypersensitivity to triheptanoin, major co-morbidities, history of severe head injury, pregnant or breastfeeding, or on tetrabenazine are not eligible.

TRIHEP3 is an international, multi-centre, randomized, double-blind, controlled, parallel phase 2 trial. It has 2 study arms: the active group, where participants receive triheptanoin oil $1 \mathrm{~g} / \mathrm{kg} / \mathrm{day}$ for 12 months; and the comparator group, where participants receive safflower oil $1 \mathrm{~g} / \mathrm{kg} /$ day for 6 months and triheptanoin oil $1 \mathrm{~g} / \mathrm{kg} /$ day for the following 6 months.

The study lasts 12 months, the first half over double-blinded conditions, and the second half as an open-label extension. Recruitment is currently closed and the study is being performed at one centre in France and one centre in the Netherlands. One hundred participants were recruited.

The primary outcomes are pharmacodynamics neuroimaging markers at 3 and 6 months $-{ }^{31} \mathrm{P}-\mathrm{MRS}$ and volumetric MRI. Secondary outcomes include the UHDRS, comprising the motor, functional and cognitive components, the Problem Behaviours Assessment Short Form, the Short Form 36 Health Survey, adverse events, tolerance and other neuroimaging biomarkers.

\section{Sponsors/funders}

Institut National de la Santé et de la Recherche Médicale and Ultragenyx Pharmaceutical Inc.

\section{Comments}

Albeit with a low success rate (15), several dietary nutrients with possible effects over metabolic processes have been tested in HD over the years, including $d-\alpha$-tocopherol (16), idebenone (17), coenzyme Q10 (18), ethyl-eicosapentaenoate (19-21), and creatine (22-24).

Triheptanoin is an odd-chain triglyceride with anaplerotic properties (i.e. it replenishes biochemical cycles with intermediate metabolites), providing the Krebs cycle with both acetyl-CoA and propionylCoA. So far, triheptanoin has been tested for several disorders of the brain metabolism, including pyruvate decarboxylase deficiency, and GLUT1 deficiency where a significant symptomatic effect was demonstrated in a small open-label study (25).

In HD, several lines of evidence support the existence of a dysfunction of the energy metabolism, including the Krebs cycle, oxidative phosphorylation and glycolysis. Two small open-label studies in HD showed that triheptanoin may have the potential to bring peripheral (26) and central nervous system metabolic biomarkers (27) to levels observed in healthy controls. They also anticipate triheptanoin to be well tolerated $(26,27)$, and overall the cumulative evidence suggests a good safety profile for doses between 1 to $2.5 \mathrm{~g} / \mathrm{kg} /$ day (14).

The TRIHEP3 trial will be completed by the end of 2019 and the results are expected in mid-2020.

\section{ACKNOWLEDGMENTS}

The authors are supported by CHDI Foundation, Inc. (salary support to FBR and EJW). 


\section{CONFLICTS OF INTEREST}

FBR and EJW were sub-investigators on LEGA TO-HD (NCT02215616), IONIS HTT Rx $_{\text {(NCT0251 }}$ 9036) and IONIS $\mathrm{HTT}_{\mathrm{Rx}}$ OLE (NCT03342053), and are sub-investigators on the Roche GENERATIONHD (NCT03761849), Roche Natural History Study (NCT03664804) and Roche GEN-EXTEND (NCT03842969) trials, and EJW was a subinvestigator on the Amaryllis (NCT02197130). EJW is the chief investigator of the Roche GEN-PEAK trial (NCT04000594) and FBR is a sub-investigator. JJF was principal investigator on LEGATO-HD and on a trial of ethyl-eicosapentanoate in Huntington's disease. The authors did not make use of confidential or privileged information: all materials included in this manuscript were collected from publicly available sources. FBR has provided consultancy services to GLG. EJW has participated in scientific advisory boards with Hoffmann-La Roche Ltd, Ionis, Shire, GSK, Wave Life Sciences, PTC Therapeutics, Takeda and Mitoconix. All honoraria were paid through UCL Consultants Ltd, a wholly owned subsidiary of UCL. Their Host Institution, University College London Hospitals NHS Foundation Trust, has received funds as compensation for conducting clinical trials for Ionis Pharmaceuticals, Pfizer and Teva Pharmaceuticals. Hoffman La Roche Ltd has supported UCL with research funding for EJW. In view of the support to both regular authors from Hoffman-La Roche Ltd, JJF was invited to be a co-author to ensure the sections on the Ionis/Roche program were suitably balanced.

JJF received grants from GlaxoSmithKline, Grunenthal, Fundação MSD (Portugal), TEVA, MSD, Allergan, Novartis, Medtronic. He received consultancy fees from GlaxoSmithKline, Novartis, TEVA, Lundbeck, Solvay, BIAL, Merck-Serono, Merz, Ipsen, Biogen, Acadia, Allergan, Abbvie, Sunovion Pharmaceuticals. He is employed by Faculdade de Medicina de Lisboa and CNS - Campus Neurológico Sénior. He also participated in advisory boards for Bial and expert testimony to Novartis.

\section{REFERENCES}

[1] Heinrich-Heine University, KKS Netzwerk, Medtronic, The George Huntington Institute, EHDN, CHDI Foundation Inc. Deep Brain Stimulation (DBS) of the Globus Pallidus (GP) in Huntington's Disease (HD). https://ClinicalTrials.gov/ show/NCT02535884; 2014.
[2] Institut National de la Santé Et de la Recherche Médicale, Ultragenyx Pharmaceutical Inc. A Comparative Phase 2 Study Assessing the Efficacy of Triheptanoin, an Anaplerotic Therapy in Huntington's Disease. https://Clinical Trials.gov/show/NCT02453061; 2015.

[3] Rodrigues FB, Wild EJ. Clinical trials corner: September 2017. J Huntingtons Dis. 2017;6(3):255-63.

[4] Tabrizi SJ, Leavitt BR, Landwehrmeyer GB, Wild EJ, Saft $\mathrm{C}$, Barker RA, et al. Targeting huntingtin expression in patients with huntington's disease. The New England Journal of Medicine. 2019.

[5] Hoffmann-La Roche. A Study to Evaluate the Efficacy and Safety of Intrathecally Administered RO7234292 (RG6042) in Patients With Manifest Huntington's Disease. https://ClinicalTrials.gov/show/NCT03761849; 2018.

[6] Ionis Pharmaceuticals I. Study in Huntington's Disease Patients Who Participated in Prior Investigational Studies of ISIS 443139. https://ClinicalTrials.gov/show/NCT 03342053; 2017.

[7] Hoffmann-La Roche. Study to Measure Cerebrospinal Fluid Mutant Huntingtin Protein in Participants With Early Manifest Stage I or Stage II Huntington's Disease. https://ClinicalTrials.gov/show/NCT03664804; 2018.

[8] Hoffmann-La Roche. An Open-Label Extension Study to Evaluate Long-Term Safety and Tolerability of RO7234292 (RG6042) in Huntington's Disease Patients Who Participated in Prior Roche and Genentech Sponsored Studies. https://ClinicalTrials.gov/show/NCT03842969; 2019.

[9] Hoffmann-La Roche. A Study to Evaluate the Safety, Tolerability, Pharmacokinetics, and Pharmacodynamics of RO7234292 (ISIS 443139) in Huntington's Disease Patients Who Participated in Prior Investigational Studies of RO7234292 (ISIS 443139). https://ClinicalTrials.gov/ show/NCT03342053; 2017.

[10] Hoffmann-La Roche. A Study to Investigate the Pharmacokinetics and Pharmacodynamics of RO7234292 (RG6042) in CSF and Plasma, and Safety and Tolerability Following Intrathecal Administration in Patients With Huntington's Disease. https://ClinicalTrials.gov/show/NCT 04000594; 2019.

[11] Wojtecki L, Groiss SJ, Hartmann CJ, Elben S, Omlor S, Schnitzler A, et al. Deep brain stimulation in huntington's disease-preliminary evidence on pathophysiology, efficacy and safety. Brain Sci. 2016;6(3):38.

[12] Wojtecki L, Groiss SJ, Ferrea S, Elben S, Hartmann CJ, Dunnett SB, et al. A prospective pilot trial for pallidal deep brain stimulation in huntington's disease. Front Neurol. 2015;6:177.

[13] Gonzalez V, Cif L, Biolsi B, Garcia-Ptacek S, Seychelles A, Sanrey E, et al. Deep brain stimulation for Huntington's disease: Long-term results of a prospective open-label study. Journal of Neurosurgery. 2014;121(1):114-22.

[14] Mochel F. Triheptanoin for the treatment of brain energy deficit: A 14-year experience. Journal of Neuroscience Research. 2017;95(11):2236-43.

[15] Travessa AM, Rodrigues FB, Mestre TA, Ferreira JJ. Fifteen years of clinical trials in huntington's disease: A very low clinical drug development success rate. Journal of Huntington's Disease. 2017;6(2):157-63.

[16] Peyser CE, Folstein M, Chase GA, Starkstein S, Brandt J, Cockrell JR, et al. Trial of d-alpha-tocopherol in Huntington's disease. Am J Psychiatry. 1995;152(12):1771-5.

[17] Ranen NG, Peyser CE, Coyle JT, Bylsma FW, Sherr M, Day L, et al. A controlled trial of idebenone in Hunting- 
ton's disease. Movement Disorders: Official Journal of the Movement Disorder Society. 1996;11(5):549-54.

[18] Huntington Study Group. A randomized, placebocontrolled trial of coenzyme Q10 and remacemide in Huntington's disease. Neurology. 2001;57(3):397-404.

[19] Puri BK, Leavitt BR, Hayden MR, Ross CA, Rosenblatt A, Greenamyre JT, et al. Ethyl-EPA in Huntington disease: A double-blind, randomized, placebo-controlled trial. Neurology. 2005;65(2):286-92.

[20] Huntington Study Group T-HDI, Dorsey ER, Shoulson I, Leavitt B, Ross C, Beck CA, et al. Randomized controlled trial of ethyl-eicosapentaenoic acid in Huntington disease: The TREND-HD study. Archives of Neurology. 2008;65(12):1582-9.

[21] Ferreira JJ, Rosser A, Craufurd D, Squitieri F, Mallard N, Landwehrmeyer B. Ethyl-eicosapentaenoic acid treatment in Huntington's disease: A placebo-controlled clinical trial. Movement Disorders: Official Journal of the Movement Disorder Society. 2015;30(10):1426-9.

[22] Rosas HD, Doros G, Gevorkian S, Malarick K, Reuter M, Coutu JP, et al. PRECREST: A phase II prevention and biomarker trial of creatine in at-risk Huntington disease. Neurology. 2014;82(10):850-7.

[23] Hersch SM, Schifitto G, Oakes D, Bredlau A-L, Meyers CM, Nahin R, et al. The CREST-E study of creatine for Huntington disease: A randomized controlled trial. Neurology. 2017;89(6):594-601.
[24] Hersch SM, Gevorkian S, Marder K, Moskowitz C, Feigin A, Cox M, et al. Creatine in Huntington disease is safe, tolerable, bioavailable in brain and reduces serum $8 \mathrm{OH} 2$ 'dG. Neurology. 2006;66(2):250-2.

[25] Mochel F, Hainque E, Gras D, Adanyeguh IM, Caillet S, Heron B, et al. Triheptanoin dramatically reduces paroxysmal motor disorder in patients with GLUT1 deficiency. J Neurol Neurosurg Psychiatry. 2016;87(5):550-3.

[26] Mochel F, Duteil S, Marelli C, Jauffret C, Barles A, Holm $\mathrm{J}$, et al. Dietary anaplerotic therapy improves peripheral tissue energy metabolism in patients with Huntington's disease. European Journal of Human Genetics: EJHG. 2010;18(9):1057-60.

[27] Adanyeguh IM, Rinaldi D, Henry PG, Caillet S, Valabregue R, Durr A, et al. Triheptanoin improves brain energy metabolism in patients with Huntington disease. Neurology. 2015;84(5):490-5.

[28] Rodrigues FB, Wild EJ. Huntingtons disease clinical trials corner: February 2018. Journal of Huntington's Disease. 2018;7(1):89-98.

[29] Rodrigues FB, Wild EJ. Huntington's disease clinical trials corner: August 2018. J Huntingtons Dis. 2018;7(3):279-86.

[30] Rodrigues FB, Quinn L, Wild EJ. Huntington's disease clinical trials corner: January 2019. Journal of Huntington's Disease. 2019;8(1):115-25. 